\title{
The Existence of Positive Solutions for Third-Order $p$-Laplacian $m$-Point Boundary Value Problems with Sign Changing Nonlinearity on Time Scales
}

\author{
Fuyi Xu and Zhaowei Meng \\ School of Science, Shandong University of Technology, Zibo, Shandong 255049, China
}

Correspondence should be addressed to Fuyi Xu, xfy_02@163.com

Received 25 February 2009; Revised 10 April 2009; Accepted 2 June 2009

Recommended by Alberto Cabada

We study the following third-order $p$-Laplacian $m$-point boundary value problems on time scales $\left(\phi_{p}\left(u^{\Delta \nabla}\right)\right)^{\nabla}+a(t) f(t, u(t))=0, t \in[0, T]_{\mathrm{T}^{\kappa}}, u(0)=\sum_{i=1}^{m-2} b_{i} u\left(\xi_{i}\right), u^{\Delta}(T)=0, \phi_{p}\left(u^{\Delta \nabla}(0)\right)=$ $\sum_{i=1}^{m-2} c_{i} \phi_{p}\left(u^{\Delta \nabla}\left(\xi_{i}\right)\right)$, where $\phi_{p}(s)$ is $p$-Laplacian operator, that is, $\phi_{p}(s)=|s|^{p-2} s, p>1, \phi_{p}^{-1}=$ $\phi_{q}, 1 / p+1 / q=1,0<\xi_{1}<\cdots<\xi_{m-2}<\rho(T)$. We obtain the existence of positive solutions by using fixed-point theorem in cones. In particular, the nonlinear term $f(t, u)$ is allowed to change sign. The conclusions in this paper essentially extend and improve the known results.

Copyright (C) 2009 F. Xu and Z. Meng. This is an open access article distributed under the Creative Commons Attribution License, which permits unrestricted use, distribution, and reproduction in any medium, provided the original work is properly cited.

\section{Introduction}

The theory of time scales was initiated by Hilger [1] as a mean of unifying and extending theories from differential and difference equations. The study of time scales has lead to several important applications in the study of insect population models, neural networks, heat transfer, and epidemic models, see, for example [2-6]. Recently, the boundary value problems with $p$-Laplacian operator have also been discussed extensively in literature; for example, see [7-18]. However, to the best of our knowledge, there are not many results concerning the higher-order $p$-Laplacian mutilpoint boundary value problem on time scales.

A time scale $\mathbf{T}$ is a nonempty closed subset of $R$. We make the blanket assumption that $0, T$ are points in $\mathbf{T}$. By an interval $(0, T)_{\mathrm{T}}$, we always mean the intersection of the real interval $(0, T)$ with the given time scale; that is $(0, T) \cap \mathbf{T}$.

In [19], Anderson considered the following third-order nonlinear boundary value problem (BVP):

$$
\begin{gathered}
x^{\prime \prime \prime}(t)=f(t, x(t)), \quad t_{1} \leq t \leq t_{3} \\
x\left(t_{1}\right)=x^{\prime}\left(t_{2}\right)=0, \quad \gamma x\left(t_{3}\right)+\delta x^{\prime \prime}\left(t_{3}\right)=0 .
\end{gathered}
$$


author studied the existence of solutions for the nonlinear boundary value problem by using Krasnoselskii's fixed point theorem and Leggett and Williams fixed point theorem, respectively.

In $[9,10]$, He considered the existence of positive solutions of the $p$-Laplacian dynamic equations on time scales

$$
\left(\phi_{p}\left(u^{\Delta}\right)\right)^{\nabla}+a(t) f(u(t))=0, \quad t \in[0, T]_{\mathrm{T}}
$$

satisfying the boundary conditions

$$
u(0)-B_{0}\left(u^{\Delta}(\eta)\right)=0, \quad u^{\Delta}(T)=0,
$$

or

$$
u^{\Delta}(0)=0, \quad u(T)-B_{1}\left(u^{\Delta}(\eta)\right)=0,
$$

where $\eta \in(0, \rho(T))$. He obtained the existence of at least double and triple positive solutions of the problems by using a new double fixed point theorem and triple fixed point theorem, respectively.

In [18], Zhou and Ma firstly studied the existence and iteration of positive solutions for the following third-order generalized right-focal boundary value problem with $p$-Laplacian operator

$$
\begin{gathered}
\left(\phi_{p}\left(u^{\prime \prime}\right)\right)^{\prime}(t)=q(t) f(t, u(t)), \quad 0 \leq t \leq 1, \\
u(0)=\sum_{i=1}^{m} \alpha_{i} u\left(\xi_{i}\right), \quad u^{\prime}(n)=0, \quad u^{\prime \prime}(1)=\sum_{i=1}^{n} \beta_{i} u^{\prime \prime}\left(\theta_{i}\right) .
\end{gathered}
$$

They established a corresponding iterative scheme for the problem by using the monotone iterative technique.

All the above works were done under the assumption that the nonlinear term is nonnegative. The key conditions used in the above papers ensure that positive solution is concave down. If the nonlinearity is negative somewhere, then the solution needs no longer to be concave down. As a result, it is difficult to find positive solutions of the $p$ Laplacian equation when the nonlinearity changes sign. In particular, little work has been done on the existence of positive solutions for higher order $p$-Laplacian $m$-point boundary value problems with nonlinearity $f$ being nonnegative on time scales. Therefore, it is a natural problem to consider the existence of positive solution for higher order $p$-Laplacian equations with sign changing nonlinearity on time scales. This paper attempts to fill this gap in literature. 
In this paper, by using different method, we are concerned with the existence of positive solutions for the following third-order $p$-Laplacian $m$-point boundary value problems on time scales:

$$
\begin{gathered}
\left(\phi_{p}\left(u^{\Delta \nabla}\right)\right)^{\nabla}+a(t) f(t, u(t))=0, \quad t \in[0, T]_{\mathrm{T}^{\kappa}} \\
u(0)=\sum_{i=1}^{m-2} b_{i} u\left(\xi_{i}\right), \quad u^{\Delta}(T)=0, \quad \phi_{p}\left(u^{\Delta \nabla}(0)\right)=\sum_{i=1}^{m-2} c_{i} \phi_{p}\left(u^{\Delta \nabla}\left(\xi_{i}\right)\right),
\end{gathered}
$$

where $\phi_{p}(s)$ is $p$-Laplacian operator, that is, $\phi_{p}(s)=|s|^{p-2} s, p>1, \phi_{p}^{-1}=\phi_{q}, 1 / p+1 / q=1$, and $b_{i}, c_{i}, a, f$ satisfy

$\left(H_{1}\right) \quad b_{i}, c_{i} \in[0,+\infty), 0<\xi_{1}<\cdots<\xi_{m-2}<\rho(T), 0<\sum_{i=1}^{m-2} b_{i}<1,0<\sum_{i=1}^{m-2} c_{i}<1 ;$

$\left(H_{2}\right) f:[0, T]_{\mathrm{T}^{\kappa}} \times[0,+\infty) \rightarrow(-\infty,+\infty)$ is continuous, $a \in C_{l d}\left([0, T]_{\mathrm{T}^{\kappa}},[0,+\infty)\right)$, and there exists $t_{0} \in[0, T)_{\mathrm{T}^{\kappa}}$ such that $a\left(t_{0}\right)>0$.

\section{Preliminaries and Lemmas}

For convenience, we list the following definitions which can be found in [1-5].

Definition 2.1. A time scale $\mathbf{T}$ is a nonempty closed subset of real numbers $R$. For $t<\sup \mathbf{T}$ and $r>\inf \mathbf{T}$, define the forward jump operator $\sigma$ and backward jump operator $\rho$, respectively, by

$$
\begin{aligned}
& \sigma(t)=\inf \{\tau \in \mathbf{T} \mid \tau>t\} \in \mathbf{T}, \\
& \rho(r)=\sup \{\tau \in \mathbf{T} \mid \tau<r\} \in \mathbf{T}
\end{aligned}
$$

for all $t, r \in \mathrm{T}$. If $\sigma(t)>t, t$ is said to be right scattered, if $\rho(r)<r, r$ is said to be left scattered; if $\sigma(t)=t, t$ is said to be right dense, and if $\rho(r)=r, r$ is said to be left dense. If $\mathbf{T}$ has a right scattered minimum $m$, define $\mathbf{T}_{k}=\mathbf{T}-\{m\}$; otherwise set $\mathbf{T}_{k}=\mathbf{T}$. If $\mathbf{T}$ has a left scattered maximum $M$, define $\mathbf{T}^{k}=\mathbf{T}-\{M\}$; otherwise set $\mathbf{T}^{k}=\mathbf{T}$.

Definition 2.2. For $f: \mathbf{T} \rightarrow R$ and $t \in \mathbf{T}^{k}$, the delta derivative of $f$ at the point $t$ is defined to be the number $f^{\Delta}(t)$ (provided that it exists), with the property that for each $\epsilon>0$, there is a neighborhood $U$ of $t$ such that

$$
\left|f(\sigma(t))-f(s)-f^{\Delta}(t)(\sigma(t)-s)\right| \leq \epsilon|\sigma(t)-s|
$$

for all $s \in U$.

For $f: \mathbf{T} \rightarrow R$ and $t \in \mathbf{T}_{k}$, the nabla derivative of $f$ at $t$, denoted by $f^{\nabla}(t)$ (provided it exists) with the property that for each $\epsilon>0$, there is a neighborhood $U$ of $t$ such that

$$
\left|f(\rho(t))-f(s)-f^{\nabla}(t)(\rho(t)-s)\right| \leq \epsilon|\rho(t)-s|
$$

for all $s \in U$. 
Definition 2.3. A function $f$ is left-dense continuous (i.e., $l d$-continuous), if $f$ is continuous at each left-dense point in $\mathbf{T}$ and its right-sided limit exists at each right-dense point in $\mathbf{T}$.

Definition 2.4. If $\phi^{\Delta}(t)=f(t)$, then we define the delta integral by

$$
\int_{a}^{b} f(t) \Delta t=\phi(b)-\phi(a)
$$

If $F^{\nabla}(t)=f(t)$, then we define the nabla integral by

$$
\int_{a}^{b} f(t) \nabla t=F(b)-F(a)
$$

Lemma 2.5. If condition $\left(H_{1}\right)$ holds, then for $h \in C_{l d}[0, T]_{T^{\kappa}}$, the boundary value problem (BVP)

$$
\begin{aligned}
& u^{\Delta \nabla}+h(t)=0, \quad t \in[0, T]_{\mathrm{T}^{\kappa}} \\
& u(0)=\sum_{i=1}^{m-2} b_{i} u\left(\xi_{i}\right), \quad u^{\Delta}(T)=0
\end{aligned}
$$

has the unique solution

$$
u(t)=\int_{0}^{t}(T-s) h(s) \nabla s+\frac{\sum_{i=1}^{m-2} b_{i} \int_{0}^{\xi_{i}}(T-s) h(s) \nabla s}{1-\sum_{i=1}^{m-2} b_{i}} .
$$

Proof. By caculating, we can easily get (2.7). So we omit it.

Lemma 2.6. If condition $\left(H_{1}\right)$ holds, then for $h \in C_{l d}[0, T]_{T^{\kappa}}$, the boundary value problem (BVP)

$$
\begin{gathered}
\left(\phi_{p}\left(u^{\Delta \nabla}\right)\right)^{\nabla}+h(t)=0, \quad t \in[0, T]_{\mathrm{T}^{\kappa}} \\
u(0)=\sum_{i=1}^{m-2} b_{i} u\left(\xi_{i}\right), \quad u^{\Delta}(T)=0, \quad \phi_{p}\left(u^{\Delta \nabla}(0)\right)=\sum_{i=1}^{m-2} c_{i} \phi_{p}\left(u^{\Delta \nabla}\left(\xi_{i}\right)\right)
\end{gathered}
$$

has the unique solution

$$
u(t)=\int_{0}^{t}(T-s) \phi_{q}\left(\int_{0}^{s} h(r) \nabla r+C\right) \nabla s+\frac{\sum_{i=1}^{m-2} b_{i} \int_{0}^{\xi_{i}}(T-s) \phi_{q}\left(\int_{0}^{s} h(r) \nabla r+C\right) \nabla s}{1-\sum_{i=1}^{m-2} b_{i}}
$$

where $C=\sum_{i=1}^{m-2} c_{i} \int_{0}^{\xi_{i}} h(r) \nabla r /\left(1-\sum_{i=1}^{m-2} c_{i}\right)$. 
Proof. Integrating both sides of equation in $(2.8)$ on $[0, t]$, we have

$$
\phi_{p}\left(u^{\Delta \nabla}(t)\right)=\phi_{p}\left(u^{\Delta \nabla}(0)\right)-\int_{0}^{t} h(r) \nabla r
$$

So,

$$
\phi_{p}\left(u^{\Delta \nabla}\left(\xi_{i}\right)\right)=\phi_{p}\left(u^{\Delta \nabla}(0)\right)-\int_{0}^{\xi_{i}} h(r) \nabla r
$$

By boundary value condition $\phi_{p}\left(u^{\Delta \nabla}(0)\right)=\sum_{i=1}^{m-2} c_{i} \phi_{p}\left(u^{\Delta \nabla}\left(\xi_{i}\right)\right)$, we have

$$
\phi_{p}\left(u^{\Delta \nabla}(0)\right)=-\frac{\sum_{i=1}^{m-2} c_{i} \int_{0}^{\xi_{i}} h(r) \nabla r}{1-\sum_{i=1}^{m-2} c_{i}}
$$

By (2.10) and (2.12) we know

$$
u^{\Delta \nabla}(t)=-\phi_{q}\left(\frac{\sum_{i=1}^{m-2} c_{i} \int_{0}^{\xi_{i}} h(r) \nabla r}{1-\sum_{i=1}^{m-2} c_{i}}+\int_{0}^{t} h(r) \nabla r\right)
$$

This together with Lemma 2.5 implies that

$$
u(t)=\int_{0}^{t}(T-s) \phi_{q}\left(\int_{0}^{s} h(r) \nabla r+C\right) \nabla s+\frac{\sum_{i=1}^{m-2} b_{i} \int_{0}^{\xi_{i}}(T-s) \phi_{q}\left(\int_{0}^{s} h(r) \nabla r+C\right) \nabla s}{1-\sum_{i=1}^{m-2} b_{i}}
$$

where $C=\sum_{i=1}^{m-2} c_{i} \int_{0}^{\xi_{i}} h(r) \nabla r /\left(1-\sum_{i=1}^{m-2} c_{i}\right)$. The proof is complete.

Lemma 2.7. Let condition $\left(H_{1}\right)$ holds If $h \in C_{l d}[0, T]_{\mathrm{T}^{\kappa}}$ and $h(t) \geq 0$, then the unique solution $u(t)$ of (2.8) satisfies

$$
u(t) \geq 0, \quad t \in[0, T]_{\mathrm{T}^{\kappa}} .
$$

Proof. By $u^{\Delta \nabla}(t)=-\phi_{q}\left(\sum_{i=1}^{m-2} c_{i} \int_{0}^{\xi_{i}} h(r) \nabla r /\left(1-\sum_{i=1}^{m-2} c_{i}\right)+\int_{0}^{t} h(r) \nabla r\right) \leq 0$, we can know that the graph of $u(t)$ is concave down on $(0, T)_{T^{*}}$, and $u^{\Delta}(t)$ is nonincreasing on $[0, T]_{T^{\kappa}}$. This together with the assumption that the boundary condition $u^{\Delta}(T)=0$ implies that $u^{\Delta}(t) \geq 0$ for $t \in[0, T]_{\mathrm{T}^{\kappa}}$. This implies that

$$
\min _{t \in[0, T]_{\mathrm{T}^{\kappa}}} u(t)=u(0)
$$


So we only prove $u(0) \geq 0$. By condition $\left(H_{1}\right)$ we have

$$
u(0)=\frac{\sum_{i=1}^{m-2} b_{i} \int_{0}^{\xi_{i}}(T-s) \phi_{q}\left(\int_{0}^{s} h(r) \nabla r+C\right) \nabla s}{1-\sum_{i=1}^{m-2} b_{i}} \geq 0 .
$$

The proof is completed.

Lemma 2.8. Let condition $\left(H_{1}\right)$ hold. If $h \in C_{l d}[0, T]_{\mathrm{T}^{x}}$ and $h(t) \geq 0$, then the unique positive solution $u(t)$ of $(B V P)(2.8)$ satisfies

$$
\inf _{t \in[0, T]_{T^{K}}} u(t) \geq \sigma_{1}\|u\|
$$

where $\sigma_{1}=\sum_{i=1}^{m-2} b_{i} \xi_{i} /\left(T-\sum_{i=1}^{m-2} b_{i}\left(T-\xi_{i}\right)\right),\|u\|=\sup _{t \in[0, T]_{\mathrm{T}}}|u(t)|$.

Proof. By $u^{\Delta \nabla}(t)=-\phi_{q}\left(\sum_{i=1}^{m-2} c_{i} \int_{0}^{\xi_{i}} h(r) \nabla r /\left(1-\sum_{i=1}^{m-2} c_{i}\right)+\int_{0}^{t} h(r) \nabla r\right) \leq 0$, we can know that the graph of $u(t)$ is concave down on $(0, T)_{\mathrm{T}^{*}}$, and $u^{\Delta}(t)$ is nonincreasing on $[0, T]_{\mathrm{T}^{\kappa}}$. This together with the assumption that the boundary condition $u^{\Delta}(T)=0$ implies that $u^{\Delta}(t) \geq 0$ for $t \in[0, T]_{\mathrm{T}^{\kappa}}$. This implies that

$$
\|u\|=u(T), \quad \min _{t \in[0, T]_{\mathrm{T}^{\kappa}}} u(t)=u(0) .
$$

For all $i \in\{1,2, \ldots, m-2\}$, we have from the concavity of $u$ that

$$
\frac{u\left(\xi_{i}\right)-u(0)}{\xi_{i}} \geq \frac{u(T)-u(0)}{T}
$$

that is,

$$
u\left(\xi_{i}\right)-u(0)+\frac{\xi_{i}}{T} u(0) \geq \frac{\xi_{i}}{T} u(T)
$$

This together with the boundary condition $u(0)=\sum_{i=1}^{m-2} b_{i} u\left(\xi_{i}\right)$ implies that

$$
\min _{t \in[0, T]_{T^{\kappa}}} u(t) \geq \frac{\sum_{i=1}^{m-2} b_{i} \xi_{i}}{T-\sum_{i=1}^{m-2} b_{i}\left(T-\xi_{i}\right)} u(T) .
$$

This completes the proof.

Let $E=C_{l d}[0, T]_{\mathrm{T}^{\kappa}}$ be endowed with the ordering $x \leq y$ if $x(t) \leq y(t)$ for all $t \in[0, T]_{\mathrm{T}^{\kappa}}$, and $\|u\|=\max _{t \in[0, T]_{T^{\kappa}}}|u(t)|$ is defined as usual by maximum norm. Clearly, it follows that $(E,\|u\|)$ is a Banach space. 
For the convenience, let

$$
\psi(s)=\phi_{q}\left(\int_{0}^{s} a(r) \nabla r+\frac{\sum_{i=1}^{m-2} c_{i} \int_{0}^{\xi_{i}} a(r) \nabla r}{1-\sum_{i=1}^{m-2} c_{i}}\right) .
$$

We define two cones by

$$
\begin{gathered}
P=\left\{u: u \in E, u(t) \geq 0, t \in(0, T)_{\mathrm{T}^{\kappa}}\right\}, \\
K=\left\{u: u \in E, u(t) \text { is concave, nonincreasing and nonnegative on }(0, T)_{\mathrm{T}^{\kappa}}\right. \\
\left.\min _{t \in[0, T]_{\mathrm{T}^{\kappa}}} u(t) \geq \sigma\|u\|\right\},
\end{gathered}
$$

where $\sigma=\sigma_{1} \sigma_{2}, \sigma_{1}$ is defined in Lemma 2.8 and

$$
\sigma_{2}=\frac{\sum_{i=1}^{m-2} b_{i} \int_{0}^{\xi_{i}} \psi(s) \nabla s}{\left(1-\sum_{i=1}^{m-2} b_{i}\right)\left(\int_{0}^{T}(T-s) \psi(T) \nabla s+\sum_{i=1}^{m-2} b_{i} \int_{0}^{\xi_{i}} \psi(T) \nabla s /\left(1-\sum_{i=1}^{m-2} b_{i}\right)\right)} .
$$

Define the operators $F: P \rightarrow E$ and $S: K \rightarrow E$ by setting

$$
\begin{aligned}
(F u)(t)= & \int_{0}^{t}(T-s) \phi_{q}\left(\int_{0}^{s} a(r) f(r, u(r)) \nabla r+A\right) \nabla s \\
& +\frac{\sum_{i=1}^{m-2} b_{i} \int_{0}^{\xi_{i}}(T-s) \phi_{q}\left(\int_{0}^{s} a(r) f(r, u(r)) \nabla r+A\right) \nabla s}{1-\sum_{i=1}^{m-2} b_{i}},
\end{aligned}
$$

where $A=\sum_{i=1}^{m-2} c_{i} \int_{0}^{\xi_{i}} a(r) f(r, u(r)) \nabla r /\left(1-\sum_{i=1}^{m-2} c_{i}\right)$,

$$
(S u)(t)=\int_{0}^{t}(T-s) \varphi(s) \nabla s+\frac{\sum_{i=1}^{m-2} b_{i} \int_{0}^{\xi_{i}} \varphi(s) \nabla s}{1-\sum_{i=1}^{m-2} b_{i}}
$$

where $\varphi(s)=\phi_{q}\left(\int_{0}^{s} a(r) f^{+}(r, u(r)) \nabla r+\tilde{A}\right), \tilde{A}=\sum_{i=1}^{m-2} c_{i} \int_{0}^{\xi_{i}} a(r) f^{+}(r, u(r)) \nabla r /\left(1-\sum_{i=1}^{m-2} c_{i}\right)$, and $f^{+}(t, u(t))=\max \{f(t, u(t)), 0\}$. Obviously, $u$ is a solution of the BVP(1.6) if and only if $u$ is a fixed point of operator $F$.

Lemma 2.9. $S: K \rightarrow K$ is completely continuous.

Proof. It is easy to see that $S K \subset K$ by $f^{+} \geq 0$ and Lemma 2.8. By Arzela-Ascoli theorem and Lebesgue dominated convergence theorem, we can easily prove that operator $S$ is completely continuous. 
Lemma 2.10 (see $[20,21])$. Let $K$ be a cone in a Banach space $X$. Let $D$ be an open bounded subset of $X$ with $D_{K}=D \cap K \neq \emptyset$ and $\bar{D}_{K} \neq K$. Assume that $A: \bar{D}_{K} \rightarrow K$ is a compact map such that $x \neq A x$ for $x \in \partial D_{K}$. Then the following results hold.

(1) If $\|A x\| \leq\|x\|, x \in \partial D_{K}$, then $i_{K}\left(A, D_{K}\right)=1$.

(2) If there exists $x_{0} \in K \backslash\{0\}$ such that $x \neq A x+\lambda x_{0}$ for all $x \in \partial D_{K}$ and all $\lambda>0$, then $i_{K}\left(A, D_{K}\right)=0$.

(3) Let $U$ be open in $X$ such that $\bar{U} \subset D_{K}$. If $i_{K}\left(A, D_{K}\right)=1$ and $i_{K}\left(A, U_{K}\right)=0$, then $A$ has a fixed point in $D_{K} \backslash \bar{U}_{K}$. The same result holds if $i_{K}\left(A, D_{K}\right)=0$ and $i_{K}\left(A, U_{K}\right)=1$, where $i_{K}\left(A, D_{K}\right)$ denotes fixed point index.

We define

$$
K_{\rho}=\{u(t) \in K:\|u\|<\rho\}, \quad \Omega_{\rho}=\left\{u(t) \in K: \min _{t \in[0, T]_{\mathrm{T}^{\kappa}}} u(t)<\sigma \rho\right\} .
$$

Lemma 2.11 (see [20]). $\Omega_{\rho}$ defined above has the following properties:

(a) $K_{\sigma \rho} \subset \Omega_{\rho} \subset K_{\rho}$;

(b) $\Omega_{\rho}$ is open relative to $K$;

(c) $u \in \partial \Omega_{\rho}$ if and only if $\min _{t \in[0, T]_{\mathrm{T}^{\kappa}}} u(t)=\sigma \rho$;

(d) if $u \in \partial \Omega_{\rho}$, then $\sigma \rho \leq u(t) \leq \rho$ for $t \in[0, T]_{\mathrm{T}^{\kappa}}$.

For the convenience, we introduce the following notations:

$$
\frac{1}{m}=\int_{0}^{T}(T-s) \psi(T) \nabla s+\frac{\sum_{i=1}^{m-2} b_{i} \int_{0}^{\xi_{i}} \psi(T) \nabla s}{1-\sum_{i=1}^{m-2} b_{i}}, \quad \frac{1}{M}=\frac{\sum_{i=1}^{m-2} b_{i} \int_{0}^{\xi_{i}} \psi(s) \nabla s}{1-\sum_{i=1}^{m-2} b_{i}} .
$$

Remark 2.12. By $\left(H_{1}\right)$ we can know that $0<m, M<+\infty, M \sigma=M \sigma_{1} \sigma_{2}=m \sigma_{1}<m$.

Lemma 2.13. If $f$ satisfies the following condition:

$$
f(t, u) \leq \phi_{p}(m \rho), \quad(t, u) \in[0, T]_{\mathrm{T}^{\kappa}} \times[0, \rho], u \neq S u, u \in \partial K_{\rho},
$$

then

$$
i_{K}\left(S, K_{\rho}\right)=1 .
$$

Proof. For $u \in \partial K_{\rho}$, then from (2.30) we have

$$
\begin{aligned}
\int_{0}^{s} a(r) f^{+}(r, u(r)) \nabla r+\tilde{A} & =\int_{0}^{s} a(r) f^{+}(r, u(r)) \nabla r+\frac{\sum_{i=1}^{m-2} c_{i} \int_{0}^{\xi_{i}} a(r) f^{+}(r, u(r)) \nabla r}{1-\sum_{i=1}^{m-2} c_{i}} \\
& \leq \phi_{p}(m \rho)\left(\int_{0}^{T} a(r) \nabla r+\frac{\sum_{i=1}^{m-2} c_{i} \int_{0}^{\xi_{i}} a(r) \nabla r}{1-\sum_{i=1}^{m-2} c_{i}}\right) .
\end{aligned}
$$


So that

$$
\varphi(s)=\phi_{q}\left(\int_{0}^{s} a(r) f^{+}(r, u(r)) \nabla r+\tilde{A}\right) \leq m \rho \psi(T) .
$$

Therefore,

$$
\begin{aligned}
S u(t) & \leq \int_{0}^{T}(T-s) \varphi(s) \nabla s+\frac{\sum_{i=1}^{m-2} b_{i} \int_{0}^{\xi_{i}} \varphi(s) \nabla s}{1-\sum_{i=1}^{m-2} b_{i}} \\
& \leq m \rho\left(\int_{0}^{T}(T-s) \psi(T) \nabla s+\frac{\sum_{i=1}^{m-2} b_{i} \int_{0}^{\xi_{i}} \psi(T) \nabla s}{1-\sum_{i=1}^{m-2} b_{i}}\right)=\rho .
\end{aligned}
$$

This implies that $\|S u\| \leq\|u\|$ for $u \in \partial K_{\rho}$. Hence by Lemma 2.10(1) it follows that $i_{K}\left(S, K_{\rho}\right)=$ 1.

Lemma 2.14. If $f$ satisfies the following condition:

$$
f(t, u) \geq \phi_{p}(M \sigma \rho), \quad(t, u) \in[0, T]_{T^{\kappa}} \times[\sigma \rho, \rho], u \neq S u, u \in \partial \Omega_{\rho},
$$

then

$$
i_{K}\left(S, \Omega_{\rho}\right)=0
$$

Proof. Let $e(t) \equiv 1$ for $t \in[0, T]_{\mathrm{T}^{\kappa}}$. Then $e \in \partial K_{1}$. We claim that

$$
u \neq S u+\lambda e, \quad u \in \partial \Omega_{\rho}, \lambda>0 .
$$

In fact, if not, there exist $u_{0} \in \partial \Omega_{\rho}$ and $\lambda_{0}>0$ such that $u_{0}=S u_{0}+\lambda_{0} e$. By $f\left(t, u_{0}\right) \geq \phi_{p}(M \sigma \rho)$, we have

$$
\begin{aligned}
\int_{0}^{s} a(r) f^{+}\left(r, u_{0}(r)\right) \nabla r+\widetilde{A} & =\int_{0}^{s} a(r) f^{+}\left(r, u_{0}(r)\right) \nabla r+\frac{\sum_{i=1}^{m-2} c_{i} \int_{0}^{\xi_{i}} a(r) f^{+}\left(r, u_{0}(r)\right) \nabla r}{1-\sum_{i=1}^{m-2} c_{i}} \\
& \geq \phi_{p}(M \sigma \rho)\left(\int_{0}^{s} a(r) \nabla r+\frac{\sum_{i=1}^{m-2} c_{i} \int_{0}^{\xi_{i}} a(r) \nabla r}{1-\sum_{i=1}^{m-2} c_{i}}\right) .
\end{aligned}
$$


So that

$$
\begin{aligned}
\varphi(s) & =\phi_{q}\left(\int_{0}^{s} a(r) f^{+}\left(r, u_{0}(r)\right) \nabla r+\tilde{A}\right) \\
& \geq \operatorname{M\sigma \rho } \phi_{q}\left(\int_{0}^{s} a(r) \nabla r+\frac{\sum_{i=1}^{m-2} c_{i} \int_{0}^{\xi_{i}} a(r) \nabla r}{1-\sum_{i=1}^{m-2} c_{i}}\right) \\
& =\operatorname{M\sigma \rho } \varphi(s) .
\end{aligned}
$$

For $t \in[0, T]_{\mathrm{T}^{\kappa}}$, then

$$
\begin{aligned}
u_{0}(t) & =S u_{0}(t)+\lambda_{0} e(t) \\
& \geq S u_{0}(0)+\lambda_{0} \\
& =\frac{\sum_{i=1}^{m-2} b_{i} \int_{0}^{\xi_{i}} \varphi(s) \nabla s}{1-\sum_{i=1}^{m-2} b_{i}}+\lambda_{0} \\
& \geq \frac{M \sigma \rho}{1-\sum_{i=1}^{m-2} b_{i}} \sum_{i=1}^{m-2} b_{i} \int_{0}^{\xi_{i}} \psi(s) \nabla s+\lambda_{0} \\
& =\sigma \rho+\lambda_{0} .
\end{aligned}
$$

This together with Lemma 2.11(c) implies that

$$
\sigma \rho \geq \sigma \rho+\lambda_{0}
$$

a contradiction. Hence by Lemma $2 \cdot 10(2)$ it follows that $i_{K}\left(S, \Omega_{\rho}\right)=0$.

\section{Main Results}

We now give our results on the existence of positive solutions of BVP (1.6).

Theorem 3.1. Suppose that conditions $\left(H_{1}\right)$ and $\left(H_{2}\right)$ hold, and assume that one of the following conditions holds.

$\left(H_{3}\right)$ There exist $\rho_{1}, \rho_{2} \in(0,+\infty)$ with $\rho_{1}<\sigma \rho_{2}$ such that

(i) $f(t, u) \leq \phi_{p}\left(m \rho_{1}\right),(t, u) \in[0, T]_{\mathrm{T}^{\kappa}} \times\left[0, \rho_{1}\right]$;

(ii) $f(t, u) \geq 0,(t, u) \in[0, T]_{\mathrm{T}^{k}} \times\left[\sigma \rho_{1}, \rho_{2}\right]$, moreover $f(t, u) \geq \phi_{p}\left(M \sigma \rho_{2}\right),(t, u) \in$ $[0, T]_{\mathrm{T}^{\kappa}} \times\left[\sigma \rho_{2}, \rho_{2}\right]$.

$\left(H_{4}\right)$ There exist $\rho_{1}, \rho_{2} \in(0,+\infty)$ with $\rho_{1}<\rho_{2}$ such that

(i) $f(t, u) \leq \phi_{p}\left(m \rho_{2}\right),(t, u) \in[0, T]_{\mathrm{T}^{\kappa}} \times\left[0, \rho_{2}\right]$;

(ii) $f(t, u) \geq \phi_{p}\left(M \sigma \rho_{1}\right),(t, u) \in[0, T]_{\mathrm{T}^{\kappa}} \times\left[\sigma^{2} \rho_{1}, \rho_{2}\right]$.

Then, the BVP (1.6) has at least one positive solution. 
Proof. Assume that $\left(H_{3}\right)$ holds, we show that $S$ has a fixed point $u_{1}$ in $\Omega_{\rho_{2}} \backslash \bar{K}_{\rho_{1}}$. By $f(t, u) \leq$ $\phi_{p}\left(m \rho_{1}\right)$ and Lemma 2.13, we have that

$$
i_{K}\left(S, K_{\rho_{1}}\right)=1
$$

By $f(t, u) \geq \phi_{p}\left(M \sigma \rho_{2}\right)$ and Lemma 2.14, we have that

$$
i_{K}\left(S, \Omega_{\rho_{2}}\right)=0
$$

By Lemma 2.11(a) and $\rho_{1}<\sigma \rho_{2}$, we have $\bar{K}_{\rho_{1}} \subset K_{\sigma \rho_{2}} \subset \Omega_{\rho_{2}}$. It follows from Lemma 2.10(3) that $S$ has a fixed point $u_{1}$ in $\Omega_{\rho_{2}} \backslash \bar{K}_{\rho_{1}}$. Clearly,

$$
\left\|u_{1}\right\|>\rho_{1}, \quad \min _{t \in[0, T]_{T^{k}}} u_{1}(t) \geq \sigma\left\|u_{1}\right\|>\sigma \rho_{1}
$$

which implies that $\sigma \rho_{1} \leq u_{1}(t) \leq \rho_{2}, t \in[0, T]_{T^{*}}$. By condition $\left(H_{3}\right)$ (ii), we have $f\left(t, u_{1}(t)\right) \geq 0$, $t \in[0, T]_{\mathrm{T}^{\kappa}}$, that is, $f^{+}\left(t, u_{1}(t)\right)=f\left(t, u_{1}(t)\right)$. Hence,

$$
F u_{1}=S u_{1}
$$

This means that $u_{1}$ is a fixed point of operator $F$.

When condition $\left(H_{4}\right)$ holds, by $f(t, u) \leq \phi_{p}\left(m \rho_{2}\right)$ and Lemma 2.13, we have that

$$
i_{K}\left(S, K_{\rho_{2}}\right)=1
$$

By $f(t, u) \geq \phi_{p}\left(M \sigma \rho_{1}\right)$ and Lemma 2.14, we have that

$$
i_{K}\left(S, \Omega_{\rho_{1}}\right)=0 .
$$

By Lemma 2.11(a) and $\rho_{1}<\rho_{2}$, we have $\bar{K}_{\sigma \rho_{1}} \subset \Omega_{\rho_{1}} \subset K_{\rho_{2}}$. It follows from Lemma 2.10(3) that $S$ has a fixed point $u_{2}$ in $K_{\rho_{2}} \backslash \bar{\Omega}_{\rho_{1}}$. Obviously,

$$
\left\|u_{2}\right\|>\sigma \rho_{1}, \min _{t \in[0, T]_{\mathrm{T}^{\kappa}}} u_{2}(t) \geq \sigma\left\|u_{2}\right\|>\sigma^{2} \rho_{1}
$$

which implies that $\sigma^{2} \rho_{1} \leq u_{2}(t) \leq \rho_{2}, t \in[0, T]_{\mathrm{T}^{\kappa}}$. By condition $\left(H_{4}\right)(\mathrm{ii})$, we have $f\left(t, u_{2}(t)\right) \geq$ $0, t \in[0, T]_{\mathrm{T}^{\kappa}}$, that is, $f^{+}\left(t, u_{2}(t)\right)=f\left(t, u_{2}(t)\right)$. Hence,

$$
F u_{2}=S u_{2}
$$

This means that $u_{2}$ is a fixed point of operator $F$. Therefore, the BVP (1.6) has at least one positive solution. 
Theorem 3.2. Assume that conditions $\left(H_{1}\right)$ and $\left(H_{2}\right)$ hold, and suppose that one of the following conditions holds.

$\left(H_{5}\right)$ There exist $\rho_{1}, \rho_{2}$, and $\rho_{3} \in(0,+\infty)$ with $\rho_{1}<\sigma \rho_{2}$, and $\rho_{2}<\rho_{3}$ such that

(i) $f(t, u) \leq \phi_{p}\left(m \rho_{1}\right),(t, u) \in[0, T]_{\mathrm{T}^{\kappa}} \times\left[0, \rho_{1}\right]$;

(ii) $f(t, u) \geq 0,(t, u) \in[0, T]_{T^{\kappa}} \times\left[\sigma \rho_{1}, \rho_{3}\right]$, moreover $f(t, u) \geq \phi_{p}\left(M \sigma \rho_{2}\right),(t, u) \in$ $[0, T]_{\mathrm{T}^{\kappa}} \times\left[\sigma \rho_{2}, \rho_{2}\right], u \neq S u, \forall u \in \partial \Omega_{\rho_{2}}$

(iii) $f(t, u) \leq \phi_{p}\left(m \rho_{3}\right),(t, u) \in[0, T]_{\mathrm{T}^{\kappa}} \times\left[0, \rho_{3}\right]$.

$\left(H_{6}\right)$ There exist $\rho_{1}, \rho_{2}$, and $\rho_{3} \in(0,+\infty)$ with $\rho_{1}<\rho_{2}<\sigma \rho_{3}$ such that

(i) $f(t, u) \geq \phi_{p}\left(M \sigma \rho_{1}\right),(t, u) \in[0, T]_{\mathrm{T}^{\kappa}} \times\left[\sigma^{2} \rho_{1}, \rho_{2}\right]$;

(ii) $f(t, u) \leq \phi_{p}\left(m_{1} \rho_{2}\right),(t, u) \in[0, T]_{\mathrm{T}^{\kappa}} \times\left[0, \rho_{2}\right], u \neq S u, \forall u \in \partial K_{\rho_{2}}$;

(iii) $f(t, u) \geq 0,(t, u) \in[0, T]_{T^{\kappa}} \times\left[\sigma \rho_{2}, \rho_{3}\right]$, moreover, $f(t, u) \geq \phi_{p}\left(M \sigma \rho_{3}\right),(t, u) \in$ $[0, T]_{\mathrm{T}^{\kappa}} \times\left[\sigma \rho_{3}, \rho_{3}\right]$.

Then, the BVP (1.6) has at least two positive solutions.

Proof. Assume that condition $\left(H_{5}\right)$ holds, we show that $S$ has a fixed point $u_{1}$ either in $\partial \mathrm{K}_{\rho_{1}}$ or in $\Omega_{\rho_{2}} \backslash \bar{K}_{\rho_{1}}$. If $u \neq S u$ for $u \in \partial K_{\rho_{1}} \cup \partial K_{\rho_{3}}$. by Lemmas 2.13 and 2.14, we have that

$$
\begin{aligned}
& i_{K}\left(S, K_{\rho_{1}}\right)=1, \\
& i_{K}\left(S, K_{\rho_{3}}\right)=1, \\
& i_{K}\left(S, \Omega_{\rho_{2}}\right)=0 .
\end{aligned}
$$

By Lemma 2.11(a) and $\rho_{1}<\sigma \rho_{2}$, we have $\bar{K}_{\rho_{1}} \subset K_{\sigma \rho_{2}} \subset \Omega_{\rho_{2}}$. It follows from Lemma 2.10(3) that $S$ has a fixed point $u_{1}$ in $\Omega_{\rho_{2}} \backslash \bar{K}_{\rho_{1}}$. Similarly, $S$ has a fixed point $u_{2}$ in $K_{\rho_{3}} \backslash \bar{\Omega}_{\rho_{2}}$. Clearly,

$$
\left\|u_{1}\right\|>\rho_{1}, \quad \min _{t \in[0, T]_{\mathrm{T}^{\kappa}}} u_{1}(t) \geq \sigma\left\|u_{1}\right\|>\sigma \rho_{1}
$$

which implies that $\sigma \rho_{1} \leq u_{1}(t) \leq \rho_{2}, t \in[0, T]_{T^{\kappa}}$. By condition $\left(H_{5}\right)\left(\right.$ ii), we have $f\left(t, u_{1}(t)\right) \geq 0$, $t \in[0, T]_{\mathrm{T}^{\kappa}}$, that is, $f^{+}\left(t, u_{1}(t)\right)=f\left(t, u_{1}(t)\right)$. Hence,

$$
F u_{1}=S u_{1}
$$

This means that $u_{1}$ is a fixed point of operator $F$. On the other hand, from $u_{2} \in K_{\rho_{3}} \backslash \bar{\Omega}_{\rho_{2}}, \rho_{2}<$ $\rho_{3}$ and Lemma 2.11(a), we have $K_{\sigma \rho_{2}} \subset \Omega_{\rho_{2}} \subset K_{\rho_{3}}$. Clearly,

$$
\left\|u_{2}\right\|>\sigma \rho_{2}, \quad \min _{t \in[0, T]_{T^{\kappa}}} u_{2}(t) \geq \sigma\left\|u_{2}\right\|>\sigma^{2} \rho_{2},
$$

which implies that $\sigma^{2} \rho_{2} \leq u_{2}(t) \leq \rho_{3}, t \in[0, T]_{T^{\kappa}}$. By $\rho_{1}<\sigma \rho_{2}$ and condition $\left(H_{5}\right)$ (ii), we have $f\left(t, u_{2}(t)\right) \geq 0, t \in[0, T]_{\mathrm{T}^{\kappa}}$, that is, $f^{+}\left(t, u_{2}(t)\right)=f\left(t, u_{2}(t)\right)$. Hence,

$$
F u_{2}=S u_{2}
$$


This means that $u_{2}$ is a fixed point of operator $F$. Then, the BVP (1.6) has at least two positive solutions.

When condition $\left(\mathrm{H}_{6}\right)$ holds, the proof is similar to the above, and so we omit it here.

\section{An Example}

In the section, we present some simple examples to explain our results.

Example 4.1. Let $\mathbf{T}=[0,1 / 2] \bigcup\{1\}, T=1$. Consider the following three-point boundary value problem with $p$-Laplacian

$$
\begin{gathered}
\left(\phi_{p}\left(u^{\Delta \nabla}\right)\right)^{\nabla}+a(t) f(t, u)=0, \quad 0<t<1, \\
u(0)=\frac{1}{3} u\left(\frac{1}{2}\right), \quad u^{\Delta}(1)=0, \quad \phi_{p}\left(u^{\Delta \nabla}(0)\right)=\frac{1}{4} \phi_{p}\left(u^{\Delta \nabla}\left(\frac{1}{2}\right)\right),
\end{gathered}
$$

where $a(t) \equiv 1, b_{1}=1 / 3, c_{1}=1 / 4, \xi_{1}=1 / 2, p=q=2$.

By computing, we can know $\sigma_{1}=1 / 5, \sigma_{2}=1 / 7, M=48 / 5, m=48 / 35$. Obviously, $\sigma=\sigma_{1} \sigma_{2}=1 / 35, M \sigma=48 / 5 \times 1 / 35<48 / 35=m$. as follows:

Let $\rho_{1}=1, \rho_{2}=78$, then $\sigma \rho_{1}<\rho_{1}<\sigma \rho_{2}<\rho_{2}$. We define a sign changing nonlinearity

$$
f(t, u)= \begin{cases}\frac{48 t^{3}}{35}\left(u-\frac{1}{35}\right)^{3}, & 0<t<1, u \in\left[0, \frac{1}{35}\right], \\ \frac{48 t^{3}}{35} \sin \left(\frac{35}{34} \frac{\pi}{2} u-\frac{1}{34} \frac{\pi}{2}\right), & 0<t<1, u \in\left[\frac{1}{35}, 1\right] \\ \frac{48 t^{3}}{35}(2-u)+\frac{3744}{175}(u-1), & 0<t<1, u \in[1,2], \\ \frac{3744}{175}+t^{3}(u-2)^{2}, & 0<t<1, u \in[2,78], \\ \frac{3744}{175}+t^{3}(78-2)^{2}[1+(u-78)], & 0<t<1, u \in[78,+\infty] .\end{cases}
$$

Then, by the definition of $f$ we have

(i) $f(t, u) \leq \phi_{p}\left(m \rho_{1}\right)=48 / 35,(t, u) \in[0,1] \times\left[0, \rho_{1}\right]$;

(ii) $f(t, u) \geq 0,(t, u) \in[0,1] \times\left[\sigma \rho_{1}, \rho_{2}\right]$, moreover $f(t, u) \geq \phi_{p}\left(M \sigma \rho_{2}\right)=3744 / 175$, $(t, u) \in[0,1] \times\left[\sigma \rho_{2}, \rho_{2}\right]$.

So condition $\left(H_{3}\right)$ holds, and by Theorem 3.1, BVP (4.1) has at least one positive solution. 


\section{Acknowledgment}

This project was supported by the National Natural Science Foundation of China (10471075, 10771117).

\section{References}

[1] S. Hilger, "Analysis on measure chains-a unified approach to continuous and discrete calculus," Results in Mathematics, vol. 18, no. 1-2, pp. 18-56, 1990.

[2] R. P. Agarwal and D. O'Regan, "Nonlinear boundary value problems on time scales," Nonlinear Analysis: Theory, Methods \& Applications, vol. 44, no. 4, pp. 527-535, 2001.

[3] F. M. Atici and G. Sh. Guseinov, "On Green's functions and positive solutions for boundary value problems on time scales," Journal of Computational and Applied Mathematics, vol. 141, no. 1-2, pp. 75-99, 2002.

[4] H.-R. Sun and W.-T. Li, "Positive solutions for nonlinear three-point boundary value problems on time scales," Journal of Mathematical Analysis and Applications, vol. 299, no. 2, pp. 508-524, 2004.

[5] M. Bohner and A. Peterso, Eds., Advances in Dynamic Equations on Time Scales, Birkhäuser, Boston, Mass, USA, 2003.

[6] H. R. Sun and W. T. Li, "Positive solutions for nonlinear $m$-point boundary value problems on time scales," Acta Mathematica Sinica, vol. 49, no. 2, pp. 369-380, 2006 (Chinese).

[7] H.-R. Sun and W.-T. Li, "Existence theory for positive solutions to one-dimensional $p$-Laplacian boundary value problems on time scales," Journal of Differential Equations, vol. 240, no. 2, pp. $217-$ 248, 2007.

[8] Y.-H. Su, W.-T. Li, and H.-R. Sun, "Triple positive pseudo-symmetric solutions of three-point BVPs for $p$-Laplacian dynamic equations on time scales," Nonlinear Analysis: Theory, Methods \& Applications, vol. 68, no. 6, pp. 1442-1452, 2008.

[9] Z. He, "Double positive solutions of three-point boundary value problems for $p$-Laplacian dynamic equations on time scales," Journal of Computational and Applied Mathematics, vol. 182, no. 2, pp. 304-315, 2005.

[10] Z. He and X. Jiang, "Triple positive solutions of boundary value problems for $p$-Laplacian dynamic equations on time scales," Journal of Mathematical Analysis and Applications, vol. 321, no. 2, pp. 911-920, 2006.

[11] F. Y. Xu, "Positive solutions for third-order nonlinear $p$-Laplacian $m$-point boundary value problems on time scales," Discrete Dynamics in Nature and Society, vol. 2008, Article ID 143040, 16 pages, 2008.

[12] Y. H. Su, S. Li, and C. Huang, "Positive solution to a singular $p$-Laplacian BVPs with sign-changing nonlinearity involving derivative on time scales," Advances in Difference Equations, vol. 2009, Article ID 623932, 21 pages, 2009.

[13] Y. H. Su and W. T. Li, "Existence of positive solutions to a singular $p$-Laplacian dynamic equations with sign changing nonlinearity," Acta Mathematica Scientia, vol. 52, pp. 181-196, 2009 (Chinese).

[14] F. Y. Xu, "Positive solutions for multipoint boundary value problems with one-dimensional $p$ Laplacian operator," Applied Mathematics and Computation, vol. 194, no. 2, pp. 366-380, 2007.

[15] Y. H. Su and W. T. Li, "Existence of positive solutions to a singular $p$-Laplacian dynamic equations with sign changing nonlinearity," Acta Mathematica Scientia, vol. 28, pp. 51-60, 2008 (Chinese).

[16] Y.-H. Su, "Multiple positive pseudo-symmetric solutions of $p$-Laplacian dynamic equations on time scales," Mathematical and Computer Modelling, vol. 49, no. 7-8, pp. 1664-1681, 2009.

[17] Y.-H. Su, W.-T. Li, and H.-R. Sun, "Positive solutions of singular $p$-Laplacian BVPs with sign changing nonlinearity on time scales," Mathematical and Computer Modelling, vol. 48, no. 5-6, pp. 845-858, 2008.

[18] C. Zhou and D. Ma, "Existence and iteration of positive solutions for a generalized right-focal boundary value problem with $p$-Laplacian operator," Journal of Mathematical Analysis and Applications, vol. 324, no. 1, pp. 409-424, 2006.

[19] D. R. Anderson, "Green's function for a third-order generalized right focal problem," Journal of Mathematical Analysis and Applications, vol. 288, no. 1, pp. 1-14, 2003.

[20] K. Q. Lan, "Multiple positive solutions of semilinear differential equations with singularities," Journal of the London Mathematical Society, vol. 63, no. 3, pp. 690-704, 2001.

[21] D. J. Guo and V. Lakshmikantham, Nonlinear Problems in Abstract Cones, vol. 5 of Notes and Reports in Mathematics in Science and Engineering, Academic Press, Boston, Mass, USA, 1988. 